\section{Screening de trastornos de estrés postraumático en población afectada por el terremoto chileno de 2010}

\author{
Screening for posttraumatic stress disorder in \\ people affected by the 2010 earthquake in Chile \\ Triagem para transtorno de estresse pós- \\ traumático em pessoas afetadas pelo terremoto \\ chileno de 2010
}

\author{
1 Escuela de Salud Pública, \\ Universidad Nacional de \\ Córdoba, Córdoba, Argentina. \\ Correspondencia \\ R. A. Abeldaño \\ Escuela de Salud Pública, \\ Universidad Nacional de \\ Córdoba. \\ Enrique Barros esq. Gordillo \\ Gómez Ciudad Universitaria 5 \\ Córdoba 5000, Argentina. \\ abeldano@arnet.com.ar
}

\begin{abstract}
The authors conducted an assessment of posttraumatic stress disorder screening in the Chilean population following the February 2010 earthquake, based on the Post Earthquake Survey with a multistage sample of 24,982 individuals over 18 years of age, applying the Davidson Trauma Scale. Multivariate analysis was performed with significance set at $p<0.05$. Prevalence of positive screening for post-traumatic stress disorder was $11 \%$ for the country as a whole, but reached $30 \%$ at lower-level disaggregation. The logistic regression model for posttraumatic stress disorder identified the following risk factors: belonging to a low-income family having suffered damage to the household, a history of health problems in the previous month, and female gender $(p<0.05)$. Family coping with the earthquake proved to be a protective factor as compared to other social or neighborhood groups, and more years of schooling were also protective $(p<0.05)$. Positive screening for posttraumatic stress disorders revealed clear social inequalities.
\end{abstract}

Post-traumatic Stress Disorders; Earthquakes; Social Inequity
Roberto Ariel Abeldaño 1

Alicia Ruth Fernández 1

Juan Carlos Estario 1

Julio Enrique Enders 1

María Josefina López de Neira ${ }^{1}$

\section{Resumen}

Se analizó un screening de trastornos de estrés postraumático en habitantes chilenos, como consecuencia del terremoto de febrero de 2010; a partir de una Encuesta Post Terremoto con una muestra multietápica de 24.982 personas mayores de 18 años, a quienes se aplicó la Escala de Trauma de Davidson. La prevalencia de screening positivo para los trastornos de estrés postraumático fue del $11 \%$ en el país, pero a menor nivel de desagregación llegaron a observarse prevalencias del 30\%. El modelo de regresión logística para la estimación de la variable trastornos de estrés postraumático identificó como factores de riesgo el hecho de pertenecer un hogar pobre, haber sufrido daños en la vivienda, haber tenido algún problema de salud en el último mes y ser mujer $(p<0,05)$. También se encontró que el afrontamiento del sismo en familia resultó como un factor protector, en relación a afrontarlo con otros colectivos sociales (vecinos), la mayor cantidad de años de educación formal también fue identificada como un factor de protección $(p<0,05)$. Se observaron claras desigualdades sociales en las personas que presentaron un screening positivo.

Trastornos de Estrés Postraumático; Terremotos; Inequidad Social 


\section{Introducción}

Como consecuencia de sus características físicoambientales, la población de Chile ha sido afectada de manera recurrente por diversos eventos naturales a lo largo de su historia, asociados a sismos y erupciones volcánicas, provocando grandes impactos en el país 1 .

El 27 de febrero de 2010 a las 3:30 AM hora local, ocurrió en Chile un terremoto de una magnitud de 8,3 grados de acuerdo con el servicio sismológico chileno, y de 8,8 grados de acuerdo al servicio sismológico estadounidense, y tuvo una duración de 2 minutos y 45 segundos. El terremoto ocurrió en el límite entre las placas tectónicas de Nazca y de Sudamérica, deslizándose la primera por debajo de la segunda placa. El epicentro estuvo localizado en la zona costera, aproximadamente a 8 kilómetros al este de la localidad de Curanipe, y a 115 kilómetros al noreste de la segunda ciudad más grande de Chile: Concepción; y pudo sentirse en varios países del cono sur: Perú, Argentina y Brasil. Luego del terremoto, se generó un tsunami que golpeó en la costa chilena, destruyendo algunas poblaciones que previamente habían sido impactadas por el movimiento telúrico, en este sentido el archipiélago Juan Fernández fue completamente devastado por la confluencia de ambos eventos 2 . Se calculó que este evento fue 31 veces más fuerte y liberó 178 veces más energía que el terremoto de Haití, ocurrido un mes atrás en el mismo año. De esa manera, este evento fue el segundo de mayor intensidad en la historia chilena, superado únicamente por el terremoto de la ciudad deValdivia en el año 1960, y uno de los 5 de mayor intensidad documentados en la historia 3 .

Las regiones más severamente afectadas por el evento fueron O’Higgins, Maule y Biobío; mientras que Valparaíso, el área Metropolitana de Santiago y Araucanía, en las cuales residían más de 13 millones de personas, es decir el $80 \%$ de la población total de Chile, también fueron zonas afectadas. De acuerdo a los reportes oficiales emitidos por la Coordinación de Asuntos Humanitarios de Naciones Unidas, y basándose en los informes del Ministerio del Interior de Chile, la cifra total de defunciones causadas por el desastre fue de 507, mientras que 441.000 viviendas fueron dañadas o destruidas. Días después del terremoto, las autoridades nacionales declararon el estado de desastre para las regiones de Libertador B. O'Higgins, Maule y Biobío para poder derivar hacia esas zonas medidas excepcionales de asistencia 4 .

A partir del evento descripto, investigadores chilenos evaluaron los trastornos psicopatológicos post-desastres, y en particular de los tras- tornos por estrés postraumático y depresivos, concluyendo que el impacto psicológico de los desastres depende tanto de las pérdidas, daños y sentimientos de amenaza que generan sobre las personas y su entorno, como de las consecuencias a largo alcance que tienen para sus vidas, en donde algunas dimensiones sociales y políticas tiene una importancia relevante 5 . Otro estudio trabajó con una muestra de habitantes de diversas localidades, en quienes evaluaron la presencia de síntomas de ataque de pánico dentro de las dos semanas posteriores a la catástrofe. Los resultados evidenciaron síntomas de crisis de pánico en personas que sufrieron pérdidas de bienes y en aquellos que estuvieron expuestos al tsunami o riesgo de tsunami 6 .

Otros autores realizaron una evaluación del estrés a posteriori de ocurrido el evento en una pequeña muestra de trabajadores de una empresa, concluyendo que si bien hubo presencia de trastornos de estrés postraumático, la satisfacción laboral fue relevante como estrategia de afrontamiento 7 .

En relación a las prevalencias de trastornos de estrés postraumático diversos estudios se refirieron a la cuantificación de la magnitud de esta problemática en diversas comunidades a partir de otros eventos, por ejemplo en Taiwán, en una muestra de personas expuestas al terremoto del año 1999 en Chi-Chi, la prevalencia de trastornos de estrés postraumático reportada fue de $4,4 \%$ a tres años del sismo 8. En Grecia 9, la prevalencia de esos trastornos a tres meses del terremoto de Ano Liosia, en el año 1999, fue de 4,5\% en una muestra de estudiantes. En California 10, se documentó una prevalencia de trastornos de estrés postraumático del $13 \%$ a tres meses del terremoto de Northridge (año 1994). En Perú, a cinco meses del terremoto de Pisco, la prevalencia publicada por el trabajo de Cairo 11 fue del $25,2 \%$. Por su parte, tras el terremoto y tsunami que golpeó al sudeste asiático diversos estudios reportaron prevalencias de estrés postraumático que van desde el $13 \%$ al $70 \%$, dependiendo del tipo de población estudiada (12-15).

Pérez et al. 16 en el año 2009 identificó en Chile una prevalencia de trastornos de estrés post traumático en el 4,4\% de la población de mayores de 15 años, antes del terremoto del año 2010, por lo que en este trabajo se toma ese valor como referencia previa al desastre.

En función de lo expuesto, y dado que en la literatura no se cuenta con aportes sobre estos trastornos generados a partir del desastre descripto en esta comunidad, se considera que la evaluación de los trastornos de estrés post traumático en situaciones de desastre puede aportar elementos útiles para la formulación de mejores 
estrategias para evitar o minimizar los efectos que producen los desastres sobre la salud mental de la población. Fueron objetivos de este trabajo: determinar la prevalencia de screening positivo para trastornos por estrés postraumático, como consecuencia del terremoto o tsunami en la población chilena; e identificar factores de riesgo para la presencia de screening positivo de trastornos de estrés postraumático, como consecuencia del terremoto o tsunami en la población chilena.

\section{Método, población y muestra}

Se analizó una base de datos secundaria correspondiente a la Encuesta Post Terremoto (EPT2010), la cual fue aplicada por parte del Ministerio de Planificación (Mideplan) en los meses de mayo y junio de 2010, es decir, a los tres meses de ocurrido el terremoto/tsunami de febrero del mismo año sobre la población chilena. La EPT recolectó datos de 22.456 hogares, que correspondieron a un subconjunto de la población entrevistada en la Encuesta de Caracterización Socioeconómica Nacional (CASEN) realizada en 2009.

El tipo de muestreo utilizado fue estratificado y multietápico. La primera etapa la conformaron comunas urbanas y comunas rurales; mientras que la segunda etapa la conformaron las secciones y dentro de cada sección se encuestaron a todos los hogares encuestados previamente en la CASEN 2009. La estratificación utilizada fue de tipo geográfico. El país se dividió en estratos, entendiendo como tal a la conjunción de división político-administrativa región o provincia en las regiones de Valparaíso, Metropolitana, Libertador B. O'Higgins, Maule, Biobío y Araucanía y áreas geográficas urbano/rural, resultando en total 67 estratos 17 .

La muestra que se consideró en el presente trabajo corresponde a las regiones de Valparaíso, Metropolitana, Libertador B. O’Higgins, Maule, Biobío, Araucanía y resto del país, resultando de esto un número de 75.986 unidades de análisis distribuidas en esas regiones, en donde se evaluaron las siguientes dimensiones: educación, trabajo, impacto en actividades independientes, ingresos - ahorro, deudas y activos financieros, salud, estrategias de participación y capital social y vivienda.

Por su parte, para evaluar específicamente la dimensión correspondiente a los trastornos de estrés postraumático, se establecieron los siguientes criterios de inclusión: (a) tener edad mayor a 18 años y; (b) estar presente en el hogar en el momento de la entrevista.
De manera que los trastornos de estrés postraumático fueron evaluados únicamente en 24.982 personas. Los demás sujetos (51.004) quedaron excluidos del análisis de estos trastornos por ser menores de 18 años o por no estar presentes en el hogar al momento de la entrevista.

\section{Instrumento y técnica de recolección de datos}

El instrumento aplicado fue un cuestionario consensuado entre el Mideplan y el Observatorio Social de la Universidad Alberto Hurtado (OSUAH). La técnica utilizada para la recolección de datos fue la entrevista personal en cuestionario de papel, aplicada por encuestadores entrenados por funcionarios del OSUAH, organismo que tuvo a su cargo la realización de la encuesta.

Para la investigación de los síntomas de trastornos de estrés postraumático, se incluyó dentro del cuestionario la versión en español de la Escala de Trauma de Davidson validada por Bobes et al. 18,19, que mide la frecuencia y severidad de los síntomas del trastorno por estrés postraumático en los sujetos que se vieron expuestos al desastre 20 . La misma está compuesta por 17 ítems, que corresponden a los 17 síntomas de trastornos de estrés postraumático, de acuerdo con el criterio de la cuarta edición del Manual Diagnóstico y Estadístico de los Trastornos Mentales (DSM-IV). Para cada ítem se puntúa la frecuencia y severidad de los síntomas durante la semana previa. Con un puntaje máximo de 136, se considera que un punto de corte de 40 , la sensibilidad es de 0,92 y su especificidad de 0,79, con una eficiencia global de 0,83 en su poder predictivo, de acuerdo al criterio DSM-IV 21,22.

\section{Análisis estadístico}

La base de datos fue procesada a través de Stata (Stata Corp., College Station, Estados Unidos), realizando análisis descriptivos e inferenciales, estableciendo un nivel de significación de $\mathrm{p}<0,05$.

Las prevalencias de trastornos de estrés postraumático fueron calculadas sobre las personas mayores de 18 años que integraron la muestra total, ya que el instrumento de medición fue aplicado específicamente en esas personas, resultando de esto un total de 24.982 personas bajo análisis. La prevalencia fue tratada como una variable dicotómica, en donde se asumió como screening positivo de trastornos de estrés postraumático a un puntaje de 40 o mayor en la Escala de Trauma de Davidson.

Para indagar la correlación de los trastornos de estrés postraumático con la edad, se aplicó el test de correlación rho de Spearman. 
Para estimar la variable dicotómica "trastornos de estrés postraumático” se definió un modelo de regresión logística binaria para la estimación de la variable dicotómica estrés postraumático ( $\mathrm{y}=0$ screening negativo; $\mathrm{y}=1$ screening positivo), utilizando como variables independientes las mismas utilizadas en el análisis descriptivo:

- Estrategias sociales de afrontamiento: si las familias enfrentaron o no problemas con el terremoto/tsunami, y en caso de haberlos enfrentado, si esto lo hicieron con su familia o colectivamente con vecinos;

- Estado de la vivienda: con o sin presencia de daños materiales, relacionados con el terremoto/ tsunami;

- Problemas de salud: haber sufrido algún problema de salud en el último mes relacionado o no al terremoto/tsunami;

- Línea de pobreza: la estimación de la pobreza se realiza por medio del cálculo del ingreso total de los hogares, dividido por el número de personas que lo integran. El resultado así obtenido, se compara con la línea de pobreza con el propósito de establecer si el hogar en cuestión -y sus integrantes- se califica como pobre o no pobre. Los valores de la línea de pobreza para el año 2.010 fueron: CL\$63.084.

También se utilizaron en la regresión logística las variables independientes edad, sexo y años de educación. Las variables relacionadas a la intensidad estimada del sismo y distancia respecto del epicentro no fueron incluidas en estos análisis, porque fueron informadas en otro artículo 23 .

\section{Aspectos éticos}

En virtud de que la base de datos utilizada es de acceso público a través de la página web del Ministerio de Desarrollo Social (http://www.mi nisteriodesarrollosocial.gob.cl/encuesta-postterremoto/base_datos.html), dicha institución, junto con el Mideplan, han codificado cada caso de análisis con técnicas de protección de la identidad de cada individuo encuestado, que sin mermar su utilidad a efectos estadísticos, impiden deducir, ni siquiera aproximadamente, cuál es el perfil de una persona concreta (Artículo 10o de la Ley no 17.622 de creación del Sistema Estadístico Nacional Argentino; y Artículos 29 o y 30o de la Ley Orgánica de creación del Instituto Nacional de Estadísticas de Chile - Ley no 17.374).

\section{Resultados}

Un análisis descriptivo sobre la muestra final de 75.896 personas evidencia que el $48,2 \%$ fueron varones y el 51,8\% mujeres. La edad media fue de 34,48 $( \pm 21,5)$ años. La media de años de educación formal fue de 9,75 $( \pm 4,5)$ años. El 15,9\% de los hogares encuestados se encontraban en ese momento bajo la línea de la pobreza. El estado civil se distribuyó en un $62,4 \%$ de casados o en relación de pareja, mientras que el 19,5\% permanecía soltero. El promedio de hijos de los hogares fue de 3,2 . Espacialmente, el $87 \%$ de la población encuestada se distribuyó en zonas urbanas.

El 79,7\% de las personas manifestaron haber afrontado el movimiento sísmico "en familia", mientras que el $20,3 \%$ lo hizo colectivamente pero con vecinos u otros colectivos sociales. El 19\% de las personas declaró haber tenido problemas de salud en el último mes (relacionados o no al sismo/tsunami); mientras que el 33,7\% de los hogares tuvo algún daño en su vivienda.

La prevalencia general de trastornos de estrés postraumático registrada en el país fue calculada en el $11,1 \%$, pero en el momento de desagregar por regiones se observaron valores que van del $7 \%$, como es el caso de la Región Metropolitana de Santiago, hasta el $24 \%$ que se observó en la región de Libertador B. O’Higgins o el 25,5\% de la Región de Biobío, mientras que las regiones que integraron la categoría de análisis "resto del país" tuvieron una prevalencia del $4,4 \%$. A un menor nivel de desagregación geográfica, como es el nivel provincial en Chile, se observó mayor afectación en las provincias de: Concepción $(30,1 \%)$, Cardenal Caro $(29,1 \%)$, Colchagua (28,9\%), Arauco (27,5\%), Cauquenes (23,5\%). La categoría de análisis "resto del país" en este nivel de descomposición también evidenció un 4,4\% de prevalencia de trastornos de estrés postraumático (Tabla 1).

En relación al sexo, se observó que el 82,5\% de las personas que resultaron con screening positivo, según la escala utilizada para la investigación fue de sexo femenino, resultando mayor a la prevalencia del $17,5 \%$ observada en las personas de sexo masculino $(\mathrm{p}<0,001)$ (Tabla 2$)$.

La correlación de los trastornos de estrés postraumático con la edad fue muy débil entre el score obtenido en las subescalas de intensidad (Rho: 0,056; $\mathrm{p}<0,05$ ) y de frecuencia (Rho: 0,052; $\mathrm{p}<$ 0,05 ), y en la escala global (Rho: 0,$054 ; p<0,05$ ).

Finalmente, en la regresión logística binaria se identificó como factor de riesgo para tener un score de screening positivo, el hecho de afrontar el evento sísmico con otros colectivos sociales como los vecinos y no en familia. Por otra parte, fueron identificados como factores de protección el pertenecer un hogar no pobre, no haber sufrido daños en la vivienda, como consecuencia del terremoto, no haber tenido problemas de salud en el último mes y ser de sexo masculino, la mayor cantidad de años de educación formal 
Distribución de las prevalencias de screening positivo para trastornos de estrés postraumático según regiones y provincias de Chile. Encuesta Post Terremoto 2010 (EPT-2010). Mideplan, Chile.

\begin{tabular}{|c|c|c|c|}
\hline & \multicolumn{2}{|c|}{ Screening positivo } & \multirow[t]{2}{*}{ Total } \\
\hline & $\mathbf{n}$ & $\%$ & \\
\hline \multicolumn{4}{|l|}{ Regiones } \\
\hline Valparaíso & 247 & 9,1 & 2.705 \\
\hline Libertador B. O'Higgins & 316 & 24,0 & 1.315 \\
\hline Maule & 343 & 22,3 & 1.539 \\
\hline Biobío & 789 & 25,5 & 3.094 \\
\hline La Araucanía & 183 & 12,3 & 1.492 \\
\hline Región Metropolitana de Santiago & 673 & 7,0 & 9.650 \\
\hline Resto del país & 230 & 4,4 & 5.187 \\
\hline \multicolumn{4}{|l|}{ Provincias } \\
\hline Valparaíso & 129 & 8,0 & 1.606 \\
\hline Los Andes & 16 & 6,8 & 235 \\
\hline Petorca & 7 & 7,3 & 96 \\
\hline Quillota & 37 & 11,5 & 322 \\
\hline San Antonio & 39 & 17,0 & 230 \\
\hline San Felipe de Aconcagua & 18 & 8,3 & 216 \\
\hline Cachapoal & 185 & 21,4 & 864 \\
\hline Cardenal Caro & 32 & 29,1 & 110 \\
\hline Colchagua & 99 & 28,9 & 342 \\
\hline Talca & 131 & 22,8 & 574 \\
\hline Cauquenes & 31 & 25,4 & 122 \\
\hline Curicó & 91 & 20,9 & 435 \\
\hline Linares & 93 & 22,7 & 410 \\
\hline Concepción & 478 & 30,1 & 1.587 \\
\hline Arauco & 86 & 27,5 & 313 \\
\hline Biobío & 97 & 16,5 & 589 \\
\hline Nuble & 127 & 21,1 & 603 \\
\hline Cautín & 140 & 12,2 & 1.144 \\
\hline Malleco & 42 & 12,1 & 348 \\
\hline Santiago & 285 & 5,7 & 4.985 \\
\hline Cordillera & 178 & 8,9 & 1.995 \\
\hline Chacabuco & 52 & 9,5 & 548 \\
\hline Maipo & 64 & 5,2 & 1.223 \\
\hline Melipilla & 55 & 12,8 & 429 \\
\hline Talagante & 39 & 8,3 & 469 \\
\hline Resto del país & 230 & 4,4 & 5.187 \\
\hline Total & 2.781 & 11,1 & 24.982 \\
\hline
\end{tabular}

también fue identificada como un factor de protección (Tabla 3).

\section{Discusión}

La prevalencia de trastornos de estrés postraumático observada en este estudio fue de $11,1 \%$ en el país, 7\% en la Región Metropolitana de Santia- go y 25,5\% en la región de Biobío (la región más próxima al epicentro); mientras que en menores niveles de desagregación geográfica las prevalencias llegaron hasta el 30,1\%, como ocurrió en la provincia de Concepción (a 115 kilómetros del epicentro).

Los prevalencias encontradas en esta indagación son compatibles con lo observado en el año 2007 en el terremoto peruano de la ciudad de 
Pisco, en donde la prevalencia publicada por el trabajo de Cairo et al. 11 fue del 25,2\% de trastornos de estrés postraumático en población general, utilizando el Harvard Trauma Questionnaire.

Por su parte, tras el terremoto y tsunami que golpeó al sudeste asiático diversos estudios reportaron prevalencias variadas de trastornos de estrés postraumático, dependiendo del tipo de población estudiada. Así en el estudio de John et al. 13, a través de los instrumentos validados para el idioma y cultura local, fueron observadas tasas de prevalencia de hasta el $70,7 \%$ en el sur de la India, cifra superior a las relevadas en Chile. Mientras que el trabajo de Neuner et al. 24, quien estudió las prevalencias en sobrevivientes del tsunami en Sri Lanka encontró tasas del 14\% al 39\% (versus tasas entre el 5\%-8\% definidas como basales, o pre-tsunami), cifras similares a las encontradas en esta indagación.

Tabla 2

Distribución del screening de trastornos de estrés postraumático según sexo. Encuesta Post Terremoto 2010 (EPT-2010). Mideplan, Chile.

\begin{tabular}{cccc}
\hline Sexo & $\begin{array}{c}\text { Screening } \\
\text { negativo }\end{array}$ & $\begin{array}{c}\text { Screening } \\
\text { positivo }\end{array}$ & Total \\
\hline $\begin{array}{ccc}\text { Varones } \\
\mathrm{n}\end{array}$ & 7.571 & 488 & 8.059 \\
$\%$ & 34,1 & 17,5 & 32,3 \\
Mujeres & & & \\
$\mathrm{n}$ & 14.630 & 2.293 & 16.923 \\
$\%$ & 65,9 & 82,5 & 67,7 \\
Total $(p<0,001)$ & & & \\
$\mathrm{n}$ & 22.201 & 2.781 & 24.982 \\
$\%$ & 100,0 & 100,0 & 100,0 \\
\hline
\end{tabular}

Las diferencias de género, en relación a la prevalencia de trastornos de estrés postraumático encontradas en este análisis, son consistentes con otros hallazgos relacionados a diversos eventos traumáticos en Dinamarca e Islandia estudios que aplicaron el Harvard Trauma Questionnaire 25, en donde las personas más afectadas fueron mujeres con menor nivel educativo, con bajos ingresos y quienes habían perdido a algún familiar en esos eventos. En este punto debe reconocerse una limitación de este trabajo al no poder incluir como covariable (importante a tener en cuenta en la definición del modelo de regresión logística) a la pérdida de familiares o seres queridos para analizar el peso de esta variable sobre el screening de síntomas de trastornos mentales, en virtud de que esta variable no fue incluida en el cuestionario aplicado por las autoridades chilenas en la EPT-2010.

$\mathrm{Al}$ confrontar estos resultados con hallazgos de otros autores que estudiaron otros terremotos, como el sismo ocurrido en el año 2008 en la localidad de Wenchuan, China 26,27, en donde se utilizaron múltiples escalas para el screening de estrés postraumático, medición del nivel de exposición y tipo de personalidad de los individuos encuestados; también se encuentra congruencia con la carga de mayor riesgo en las mujeres. Sin embargo, en esos estudios la variable edad evidenció mayor correlación con los trastornos de estrés postraumático que la observada en la presente investigación.

En el caso del desastre generado por el huracán Katrina, los diferenciales de género también fueron estudiados en Estados Unidos, concordando en que las mujeres son más vulnerables al estrés postraumático que los varones 28 . Otros autores afirman que las mujeres son más propensas que los hombres a desarrollar trastornos de estrés postraumático y que estas diferencias

Tabla 3

Modelo de regresión logística binaria para la variable dependiente "screening de los trastornos de estrés postraumático" ( $y=0$ screening negativo; $y=1$ screening positivo). Encuesta Post Terremoto 2010 (EPT-2010). Mideplan, Chile.

\begin{tabular}{|c|c|c|c|c|}
\hline \multirow{2}{*}{$\begin{array}{l}\text { Variables regresoras } \\
\text { Estrategias sociales de afrontamiento (otros colectivos sociales no-familia) }\end{array}$} & \multirow{2}{*}{ OR } & \multicolumn{2}{|c|}{ IC95\% } & \multirow{2}{*}{$\begin{array}{c}\text { Valor de } p \\
0,001\end{array}$} \\
\hline & & 1,09 & 1,46 & \\
\hline Estado de la vivienda (sin daños) & 0,42 & 0,37 & 0,48 & 0,001 \\
\hline Sin problemas de salud (último mes) & 0,50 & 0,44 & 0,58 & 0,001 \\
\hline Edad & 0,99 & 0,99 & 1,00 & 0,151 \\
\hline Sexo (varón) & 0,50 & 0,44 & 0,56 & 0,001 \\
\hline Línea de pobreza (hogar no pobre) & 0,80 & 0,68 & 0,93 & 0,005 \\
\hline Años de educación & 0,97 & 0,95 & 0,98 & 0,001 \\
\hline
\end{tabular}

IC95\%: intervalo de confianza del 95\%; OR: odds ratio. 
pueden atribuirse no sólo al orden biológico, sino también a las diferencias entre hombres y mujeres en la socialización y a las experiencias formativas de la niñez 29. Asimismo, el incremento de vulnerabilidad que experimentan las mujeres, respecto de los varones, ante la mayoría de los desastres naturales, podría estar influenciado por factores individuales representados por las habilidades de afrontamiento y de los rasgos de personalidad, y por factores del contexto social y de problemáticas de género que se reproducen y se profundizan en situaciones críticas como los desastres naturales. Estas diferencias, sumadas a los niveles de exposición al desastre pueden ser determinantes para la presencia de síntomas de depresión, ansiedad y trastornos de estrés postraumático en las mujeres 30 .

Estas diferencias que pueden atribuirse a los mandatos culturales y a los roles sociales que las mujeres desarrollen dentro de los entornos sociales y étnicos en donde viven, en muchas comunidades colocan a las mujeres en un plano de vulnerabilidad 31 , en relación a esto se puede hipotetizar que estas diferencias son particularmente evidentes en comunidades que enfatizan los roles tradicionales de género ${ }^{32}$. También se ha sugerido que el aumento de la sintomatología en las mujeres es probable que sea resultado de la exposición a diferentes factores de estrés de base en el día a día 33. Otros autores argumentaron que el mayor grado de afectividad negativa en las mujeres puede resultar en respuestas emocionales y somáticas más reactivas en las mujeres en comparación con los varones 34. A pesar de conocer todos estos argumentos, esta situación de vulnerabilidad señalada en las mujeres se ha demostrado desde hace décadas y aún no ha sido posible corregirla.

Por su parte, en relación al rol de la familia en la resiliencia, es importante destacar que la promoción de la resiliencia recae fundamentalmente sobre la familia, ya que es el principal ámbito de vinculación afectiva y de formación del estilo relacional de fortaleza o debilidad ante los eventos traumáticos 35 .

Investigadores que estudiaron trastornos mentales relacionados al tsunami del sudeste asiático, en donde la prevalencia de trastornos de estrés postraumático fue mayor a la observada en los habitantes chilenos, concuerdan con identificar como de factores de riesgo: la situación de pobreza, el sexo femenino y el hecho de haber tenido problemas de salud durante el desastre 36 . Profundizando la discusión en las variables cercanas al plano social y económico, los hallazgos del presente estudio son consistentes con lo observado en el trabajo conducido por Tracy et al. 37 , quien estudió a la población del estado de
Texas (Estados Unidos), a posteriori del huracán Ike que afectó la costa Texana en el año 2008. Allí indagaron la influencia de algunos determinantes sociales y económicos como predictores de la aparición de síntomas de trastornos mentales. Ellos demostraron el papel que juegan la educación formal, los ingresos económicos del hogar y las pérdidas materiales sufridas en el evento, sobre el diagnóstico de trastornos mentales detonados por el huracán Ike. La mayor cantidad de años de educación formal y la mayor suma de ingresos del hogar resultaron situaciones protectoras para la población; mientras que la pérdida de bienes materiales resultó un factor de riesgo para la presencia de trastornos de estrés postraumático. En ese sentido, la influencia de esos factores sociales y económicos sobre la salud mental comunitaria fueron ampliamente descriptos y destacados por diversos autores 38,39,40,41.

Esto pone de relieve una vez más que las circunstancias sociales y económicas deficientes juegan un rol importante y afectan la salud mental de las personas, especialmente en situaciones de desastre, en otras palabras los gradientes sociales se traducen también en el aumento de la prevalencia y en la aparición de algunos factores de riesgo para el aumento de la morbilidad mental. Por ello, las políticas sanitarias que busquen la protección de la salud mental deberían apuntar hacia la reducción de las desigualdades sociales para reducir sus efectos consecuentes. El modelo de análisis de regresión logística destacó algunos factores de riesgo de carácter individual, como también algunos otros relacionados con la familia y sociedad, y otro de carácter económico; desde aquí debe interpretarse que la interacción de esas situaciones de riesgo y su acumulación individual y familiar pasan a ser los verdaderos determinantes sociales que predisponen a los individuos a experimentar alguna condición mental no saludable, reflotando de alguna manera la importancia de las cuestiones sociales en el estudio de la salud mental.

Existen algunas limitaciones de este trabajo, en primer lugar, el período post-terremoto de cuatro meses fue relativamente corto y no podría concluirse que las prevalencias medidas allí sigan siendo las mismas en la actualidad, debido a la dinámica que inscriben estas condiciones; tampoco puede afirmarse que las covariables del modelo de regresión continúen mostrando significativamente los mismos ratios durante tanto tiempo después de la catástrofe; de ahí la importancia de efectuar mediciones de seguimiento. En segundo lugar, como se mencionó en párrafos anteriores, no se pudo incluir como covariable la pérdida de vidas, debido a que esta variable no fue incluida en la EPT-2010. En tercer 
lugar, el Servicio Sismológico Chileno informó de la ocurrencia de numerosos movimientos sísmicos a lo largo de todo el territorio chileno en los días posteriores al 27 de febrero, lo cual pudo haber influido en las estimaciones aquí descritas. En cuarto lugar, la versión aplicada en este estudio de la Escala de Trauma de Davidson se trata de una versión que aún no está validada en población chilena, por lo cual entre sus limitaciones deben considerarse sus diferencias transculturales.

\section{Resumo}

Foram analisados dados associados a transtornos de estresse pós-traumáticos apresentados por uта população do Chile, após o terremoto de fevereiro de 2010, a partir de uma pesquisa pós-terremoto (EPT 2010, Chile) com uma amostra multiestágio de 24.982 pessoas de mais de 18 anos, com aplicação da Escala de Trauma Davidson. A prevalência de casos positivos de screening para transtornos avaliados foi de $11 \%$ no país, mas em um nivel inferior de desagregação, a prevalência observada atingiu $30 \%$. O modelo de regressão logística para estimar a variável transtornos de estresse pós-traumático identificou como fatores de risco o fato de pertencer a uma família pobre, ter sofrido danos em seu domicílio, ter problemas de saúde no último mês e ser do sexo feminino $(p<0,05)$. Também se encontrou que enfrentar o terremoto em família resultou como um fator de proteção em comparação com outros grupos sociais ou vizinhos, o maior número de anos de educação formal também foi identificado como um fator de proteção ( $p$ $<0,05)$. Foram observadas claras desigualdades sociais em pessoas que tiveram screening positivo.

Transtornos de Estresse Pós-Traumáticos; Terremotos; Iniquidade Social

\section{Conclusiones}

En este trabajo se destaca que en las estrategias de participación social para el afrontamiento de la situación de desastre resultó beneficioso el vínculo familiar, frente al vínculo con otros colectivos sociales, enfatizando el rol de la familia en la resiliencia ante el evento. Los factores de riesgo detectados para el screening positivo de trastornos de estrés postraumático se relacionaron a la presencia de problemas de salud relacionados o no al sismo/tsunami; a pertenecer a un hogar pobre; a haber sufrido daños o destrucción en la vivienda familiar; y ser de sexo femenino. Se observaron claras desigualdades tanto sociales, como económicas, en las personas que presentaron un screening positivo para los trastornos por estrés postraumático medido a través de la Escala de Trauma de Davidson.

\section{Colaboradores}

R. A. Abeldaño participó de la ejecución del proyecto, análisis de datos, redacción del documento y revisión final. A. R. Fernández colaboró en la redacción del documento y en la revisión final. J. C. Estario y J. E. Enders participaron en la redacción del documento y revisión final. M. J. L. Neira colaboró en la revisión final del documento.

\section{Agradecimientos}

Esta investigación utilizó información de la Encuesta Post Terremoto 2010 (EPT-2010, Chile). El autor agradece al Mideplan, propietario intelectual de la encuesta, haberle permitido disponer de la base de datos. Todos los resultados del estudio son de responsabilidad del autor y en nada comprometerán a dicho Ministerio. 


\section{Referencias}

1. Comisión Económica para América Latina. Reco mendaciones para una estrategia de reconstrucción y recuperación del terremoto de Chile del 27 de febrero de 2010. Santiago: Comisión Económica para América Latina; 2010.

2. Organización Panamericana de la Salud. El terremoto y tsunami del 27 de febrero en Chile: crónica y lecciones aprendidas en el sector salud. Santiago: Organización Panamericana de la Salud; 2010.

3. Organización Panamericana de la Salud. Protección de la salud mental luego del terremoto y tsunami del 27 de febrero de 2010 en Chile: crónica de una experiencia. Santiago: Organización Panamericana de la Salud; 2010.

4. Comisión Económica para América Latina. The Chilean earthquake of 27 February 2010: an overview. Santiago: Comisión Económica para América Latina; 2010.

5. Cova F, Rincón P. El terremoto y tsunami del 27-F y sus efectos en la salud mental. Ter Psicol 2010; 28:179-85.

6. Leiva M, Quintana G. Factores ambientales y psicosociales vinculados a síntomas de ataque de pánico después del terremoto y tsunami del 27 de febrero de 2010 en la zona central de Chile. Ter Psicol 2010; 28:161-7.

7. Jiménez A, Cubillos R. Estrés percibido y satisfacción laboral después del terremoto ocurrido el 27 de febrero de 2010 en la zona centro-sur de Chile. Ter Psicol 2010; 28:187-92.

8. Wu HC, Chou P, Chou FH, Su CY, Tsai KY, Ou-Yang WC, et al. Survey of quality of life and related risk factors for a Taiwanese village population 3 years post-earthquake. Aust N Z J Psychiatry 2006; 40:355-61.

9. Roussos A, Goenjian A, Steinberg A, Sotiropoulou C, Kakaki M, Kabakos C, et al. Posttraumatic stress and depressive reactions among children and adolescents after the 1999 earthquake in Ano Liosia, Greece. Am J Psychiatry 2005; 162:530-7.

10. McMillen JC, North C, Smith E. What parts of PTSD are normal: intrusion, avoidance, or arousal? Data from the northridge, California, earthquake. J Trauma Stress 2000; 13:57-79.

11. Cairo JB, Dutta S, Nawaz H. The prevalence of posttraumatic stress disorder among adult earthquake survivors in Peru. Disaster Med Public Health Prep 2010; 4:39-46.

12. Thienkrua W, Cardozo B, Chakkraband M, Guadamuz T, Pengjuntr W, Tantipiwatanaskul P, et al. Symptoms of posttraumatic stress disorder and depression among children in tsunami-affected areas in southern Thailand. JAMA 2006; 296:549-59.

13. John P, Russell S, Russell P. The prevalence of posttraumatic stress disorder among children adolescents affected by tsunami disaster in Tamil Nadu. Disaster Manag Response 2007; 5:3-7.

14. Neuner F, Schauer E, Catani C, Ruf M, Elbert T. Post-tsunami stress: a study of posttraumatic stress disorder in children living in three severely affected regions in Sri Lanka. J Trauma Stress 2006; 19:339-47.
15. Dewaraja R, Kawamura N. Trauma intensity and posttraumatic stress: implications of the tsunami experience in Sri Lanka for the management of future disasters. Int Congr Ser 2006; 1287:69-73.

16. Pérez C, Vicente B, Zlotnick C, Kohn R, Johnson, J, Saldivia S, et al. Estudio epidemiológico de sucesos traumáticos, trastorno de estrés post-traumático y otros trastornos psiquiátricos en una muestra representativa de Chile. Salud Mental 2009; 32:145-53.

17. División Social, Ministerio de Planificación. Encuesta Post Terremoto 2010: ficha técnica. Santiago: Ministerio de Planificación; 2010.

18. Bobes J, Calcedo-Barba A, García M, François M, Rico-Villademoros E, González MP, et al. Evaluación de las propiedades psicométricas de la versión española de cinco cuestionarios para la evaluación del trastorno de estrés postraumático. Actas Esp Psiquiatr 2000; 28:207-18.

19. Bobes García J, Portilla M, Bascarán Fernández M, Sáiz Martínez P, Bousoño García M. Banco de instrumentos básicos para la práctica de la psiquiatría clínica. 2a Ed. Barcelona: Ars Médica; 2002.

20. Villafañe A, Milanesio M, Marcellino C, Amodei C. La evaluación del trastorno por estrés postraumático: aproximación a las propiedades psicométricas de la Escala de Trauma de Davidson. Revista Evaluar 2003; (3):80-93.

21. Zlotnick C, Davidson J, Tracie M, Pearlstein T. Validation of the Davidson Trauma Scale in a sample of survivors of childhood sexual abuse. J Nerv Ment Dis 1996; 184: 255-7.

22. Davidson J, Book S, Colket J, Tupler L, Roth S, David $\mathrm{D}$, et al. Assessment of a new self-rating scale for post-traumatic stress disorder. Psychol Med 1997; 27:153-60.

23. Abeldaño RA, Fernández AR, Estario JC, Enders JE. Distribución espacial de los trastornos de estrés postraumático en Chile a partir del terremoto del 27-F. Rev Salud Pública 2013; 17:40-6.

24. Neuner F, Schauer E, Catani C, Ruf M, Elbert T. Post-tsunami stress: a study of posttraumatic stress disorder in children living in three severely affected regions in Sri Lanka. J Trauma Stress 2006; 19:339-47.

25. Ditlevsen DN, Elklit A. Gender, trauma type, and PTSD prevalence: a re-analysis of 18 Nordic convenience samples. Ann Gen Psichiatry 2012; 11:26.

26. Zhang Y, Ho SM. Risk factors of posttraumatic stress disorder among survivors after the 512 Wenchuan earthquake in China. PLoS One 2011; 6:e22371.

27. Gao X, Luo X. PTSD-positive screening and factors influencing the mental state in victims evacuated/ not evacuated from Wenchuan earthquake area within 1 month. Zhong Nan Da Xue Xue Bao Yi Xue Ban 2009; 34:504-9.

28. DeSalvo KB, Hyre AD, Ompad DC, Menke A, Tynes LL, Muntner P. Symptoms of posttraumatic stress disorder in a New Orleans workforce following Hurricane Katrina. J Urban Health 2007; 84:142-52. 
29. Breslau N, Anthony J. Gender differences in the sensitivity to posttraumatic stress disorder: an epidemiological study of urban young adults. J Abnorm Psychol 2007; 116:607-11.

30. Díaz C, Quintana G, Vogel E. Síntomas de depresión, ansiedad y estrés post-traumático en adolescentes siete meses después del terremoto del 27 de febrero de 2010 en Chile. Ter Psicol 2012; 30:37-43.

31. Breslau N, Davis G, Adreski P, Peterson E, Schultz L. Sex differences in post traumatic stress disorder. Arch Gen Psychiatry 1997; 54:1044-8.

32. Norris F, Foster J, Weisshaar D. The epidemiology of sex differences in PTSD across developmental, societal, and research contexts. In: Kimerling R, Ouimette P, Wolfe J, editors. Gender and PTSD. New York: The Guilford Press; 2002. p. 3-42.

33. Banyard VL, Graham-Bermann SA. Can women cope? A gender analysis of theories of coping with stress. Psychology of Women Quarterly 1993; 17:303-18.

34. Zeidner M. Gender group differences in coping with chronic terror: the Israeli scene. Sex Roles 2006; 54:297-310.

35. Moneta ME. Apego, resiliencia y vulnerabilidad a enfermar: interacciones genotipo-ambiente. Gaceta de Psiquiatría 2007; 3:321-6.

36. Kumar MS, Murhekar MV, Hutin Y, Subramanian T, Ramachandran V, Gupte MD. Prevalence of posttraumatic stress disorder in a coastal fishing village in Tamil Nadu, India, after the December 2004 tsunami. Am J Public Health 2007; 97:99-101.
37. Tracy M, Norris FH, Galea S. Differences in the determinants of posttraumatic stress disorder and depression after a mass traumatic event. Depress Anxiety 2011; 28:666-75.

38. Galea S, Resnick H, Ahern J, Gold J, Bucuvalas M, Kilpatrick D, et al. Posttraumatic stress disorder in Manhattan, New York City, after the September 11th terrorist attacks. J Urban Health 2002; 79:340-53.

39. Galea S, Tracy M, Norris F, Coffey SF. Financial and social circumstances and the incidence and course of PTSD in Mississippi during the first two years after Hurricane Katrina. J Trauma Stress 2008; 21:357-68.

40. Armenian HK, Morikawa M, Melkonian AK, Hovanesian A, Akiskal K, Akiskal HS. Risk factors for depression in the survivors of the 1988 earthquake in Armenia. J Urban Health 2002; 79:373-82.

41. Goenjian AK, Steinberg AM, Najarian LM, Fairbanks LA, Tashjian M, Pynoos RS. Prospective study of posttraumatic stress, anxiety, and depressive reactions after earthquake and political violence. Am J Psychiatry 2000; 157:911-6.

Recibido el 01/Ago/2013

Versión final presentada el 09/Mar/2014

Aprobado el 01/Abr/2014 